\title{
CAUSTIC CONSIDERATION OF LONG PLANETARY WAVE PACKET ANALYSIS IN THE CONTINUOUSLY STRATIFIED OCEAN
}

\author{
ARTHUR D. GORMAN and HUIJUN YANG
}

(Received 19 April 2000)

\begin{abstract}
The wave packet method, one form of the WKB technique, recently has been employed to investigate the evolution of long planetary wave packets in relation to the complex climate variability in the world oceans. However, such a method becomes invalid near the caustics. Here, the Lagrange manifold formalism is used to extend this analysis to include the caustic regions. We conclude that even though the wave packet method fails near the caustics, the equations derived from this method away from caustics are identical to the ones from the Lagrange manifold formalism near caustics.
\end{abstract}

2000 Mathematics Subject Classification. Primary 76B65, 76M45, 86A05.

1. Introduction. Recently, wave packet theory has been used by Yang [10, 11] in two studies to analyze the evolution of long planetary wave packets. In the first study, the evolution of long planetary wave packets in a continuously stratified ocean with exponentially decaying stratification and mean zonal current was considered. The analysis successfully explained all major features of long planetary Rossby waves recently observed in the Topex/Poseidon satellite data in the global ocean. In the second study, the evolution of three-dimensional wave packets in a subtropical gyre was studied. This analysis led to a rudimentary theory for ocean climate variability on the inter-annual to decadal time scale observed in the sea surface height changes and the ocean temperature changes and provided insight of dynamical process of complex ocean climate variability. The ocean response may undergo transition between different regimes. The transition of regime may be one more reason that the observed climate variability in the ocean is so complicated with a variety of time scales between interannual and decadal. These predictions are consistent with observations in the North Pacific and other analytic and numerical model results. These results were also further discussed in [12]. While the focus of each investigation was the development of analytical solutions, wave packet theory enabled an analysis of the structural evolution of the wave packet associated with each setting [9].

Wave packet theory is based on the WKB formalism, originally developed for water waves but primarily associated with quantum mechanics [4]. Near caustic or turning points the classical WKB approach is not valid [6], for example, physically in regimes, where the phase velocity of the wave packet coincides with the velocity of the large-scale current. A related approach that does apply in such regimes is the Lagrange manifold formalism developed by Maslov [7] and Arnol'd [1]. Here, we use 
the Lagrange manifold formalism, as adapted to geophysical models [2, 3], to study wave phenomena near caustics for those environments considered by Yang. Our purpose is to complement his results and to illustrate how the approach may be used to study the caustic curve itself. For completeness, we include a summary of the models considered and an outline of the basic algorithm.

2. Potential vorticity equation in the zonal current. The linearized nondimensional equation governing the potential vorticity in a stratified three-dimensional quasi-geostrophic baroclinic ocean for large-scale motion can be written as

$$
\left(\frac{\partial}{\partial t}+U(z) \frac{\partial}{\partial x}\right) q^{\prime}+B \frac{\partial \psi^{\prime}}{\partial x}=0
$$

where $q^{\prime}$ is the perturbation potential vorticity, defined as

$$
q^{\prime}=\frac{\partial^{2} \psi^{\prime}}{\partial x^{2}}+\frac{\partial^{2} \psi^{\prime}}{\partial y^{2}}+\frac{\partial}{\partial z}\left(\frac{1}{S} \frac{\partial \psi^{\prime}}{\partial z}\right)
$$

In these equations, $x, y$, and $z$ are eastward, northward, and upward coordinates, respectively, $B$ is the meridional gradient of the mean potential vorticity, $Q$,

$$
B=\frac{\partial Q}{\partial y}=\beta-\frac{\partial}{\partial z}\left(\frac{1}{S} \frac{\partial U}{\partial z}\right)
$$

and $S$ is the nondimensional stratification parameter, defined as

$$
S=\frac{N^{2} D^{2}}{L^{2} f_{0}^{2}} .
$$

Here, $N$ is the Brunt-Väisälä frequency; $L$ and $D$ are the horizontal and vertical characteristic scales, respectively; $f_{0}$ is the Coriolis parameter; $\beta$ is the meridional gradient of the Coriolis parameter; $U(z)$ is the mean zonal ocean current $[8,9]$.

We assume that the stratification parameter may be modeled by an exponential function of $z$ with a depth scale $H$, namely

$$
S=S_{0} e^{z / H}
$$

where $S_{0}$ is the value of the stratification parameter at $z=0$. Exponential stratification is a commonly-used, realistic model for the global subsurface ocean. We also assume that the mean zonal ocean circulation takes the form

$$
U=U_{\mathrm{s}} e^{z / 2 H} \cos (k \pi z)
$$

where $k$ is the vertical wave number of the mean zonal ocean current and $U_{\mathrm{s}}$ is the zonal mean current at the sea surface (at the top of the thermocline). A recent discussion of these assumptions is provided in [5, 10, 11].

We begin by introducing the transformation

$$
\psi^{\prime}=e^{z / 2 H} \psi
$$


which allows us to rewrite the perturbation equation (2.1) as

$$
\left(\frac{\partial}{\partial t}+U(z) \frac{\partial}{\partial x}\right) q+B \frac{\partial \psi}{\partial x}=0
$$

where

$$
q=-\frac{\psi}{4 H^{2} S}+\frac{\partial^{2} \psi}{\partial x^{2}}+\frac{1}{S} \frac{\partial^{2} \psi}{\partial z^{2}}, \quad B=\beta+\frac{1+4 H^{2} k^{2} \pi^{2}}{4 H^{2} S} U,
$$

and the perturbation is now assumed independent of $y$, the northward coordinate, for the planetary waves.

3. Lagrange manifold algorithm. Following Yang, we assume that the mean zonal current $U$, the stratification parameter $S$, and the potential vorticity parameter $B$ are slowly varying with respect to space. We rescale our independent variables

$$
\epsilon \bar{r}=\epsilon(x, z) \longrightarrow(x, z), \quad \epsilon t \longrightarrow t,
$$

where $\epsilon$ is a small parameter, for example, physically the ratio of the characteristic horizontal scale of the wave packet to the horizonal scale of the ocean basin. Then (2.8) becomes

$$
\epsilon^{3}\left(\frac{\partial}{\partial t}+U(z) \frac{\partial}{\partial x}\right)\left(\frac{\partial^{2} \psi}{\partial x^{2}}+\frac{1}{S} \frac{\partial^{2} \psi}{\partial z^{2}}-\frac{1}{\epsilon^{2} 4 H^{2} S} \psi\right)+\epsilon B \frac{\partial \psi}{\partial x}=0,
$$

where $B$ is now the rescaled $B$, that is, $B(x, z)=B(\epsilon x, \epsilon z)$. Near caustics, we assume that (3.2) has a solution of the form

$$
\psi(x, z, t)=\int A(\bar{r}, \bar{p}, t, \epsilon) e^{i \phi / \epsilon} d \bar{p} .
$$

In (3.3), $\bar{r}=(x, z)$ are coordinates and $\bar{p}=(m, l)$ are the corresponding wave-vectors. The amplitude

$$
A(\bar{r}, \bar{p}, t, \epsilon) \sim \sum_{k=0} A_{k}(\bar{r}, \bar{p}, t)\left(\frac{i}{\epsilon}\right)^{k}
$$

and its derivatives are assumed bounded and

$$
\phi(\bar{r}, t, \bar{p}, \omega)=\bar{r} \cdot \bar{p}-\omega t-\Theta(\bar{p}, \omega),
$$

where $\Theta(\bar{p}, \omega)$ may be regarded as a phase. Then substituting (3.3) into (3.2), and, following Yang, introducing into the resulting equation wave-vector, $\bar{p}$, and frequency, $\omega$,

leads to

$$
\bar{p}=\nabla \phi, \quad \omega=-\frac{\partial \phi}{\partial t}
$$

$$
\begin{aligned}
& \int\left\{\left[B m+\left(m^{2}+\frac{l^{2}}{S}+\frac{1}{4 H^{2} S}\right)(\omega-U m)\right] A\right. \\
& \quad \times i \epsilon\left[\left(2 \omega m+B-U\left(3 m^{2}-\frac{1}{4 H^{2} S}\right)\right) \frac{\partial A}{\partial x}+\frac{2}{S}(\omega-2 U l) \frac{\partial A}{\partial z}-\left(m^{2}+\frac{l^{2}}{S}+\frac{1}{4 H^{2} S}\right) \frac{\partial A}{\partial t}\right] \\
& \quad \times(i \epsilon)^{2}\left[(3 U m-\omega) \frac{\partial^{2} A}{\partial x^{2}}+\frac{3 U l^{2}-\omega}{S} \frac{\partial^{2} A}{\partial z^{2}}+2\left(m \frac{\partial^{2} A}{\partial x \partial t}+\frac{1}{S} \frac{\partial^{2} A}{\partial z \partial t}\right)\right] \\
& \left.\quad+(i \epsilon)^{3}\left[\left(\frac{\partial}{\partial t}+U \frac{\partial}{\partial x}\right)\left(\frac{\partial^{2} A}{\partial x^{2}}+\frac{1}{S} \frac{\partial^{2} A}{\partial z^{2}}\right)\right]\right\} e^{i \psi / \epsilon} d \bar{p} \sim 0 .
\end{aligned}
$$


Here, the coefficient of the $(i \epsilon)^{0}$ term is the Hamiltonian

$$
H(\bar{r}, \bar{p})=\left(m^{2}+\frac{l^{2}}{S}+\frac{1}{4 H^{2} S}\right)(\omega-U m)+B m .
$$

On the caustics, the integral is evaluated at any point $(x, z)$ using the stationary phase condition $\left[\nabla_{p} \phi=0\right]$, which obtains the Lagrange manifold

$$
\bar{r}=\nabla_{p} \Theta(\bar{p}, \omega)
$$

and turns the Hamiltonian into an eikonal equation [2, 3],

$$
\left(m^{2}+\frac{l^{2}}{S}+\frac{1}{4 H^{2} S}\right)(\omega-U m)+B m=0 .
$$

We determine the phase from Hamilton's equations. First, the equations are solved to obtain

$$
\bar{r}=\bar{r}(\gamma, \bar{\sigma}), \quad \bar{p}=\bar{p}(\gamma, \bar{\sigma}), \quad t=t(\gamma, \bar{\sigma}), \quad \omega=\omega(\gamma, \bar{\sigma}),
$$

where $\gamma$ is an arbitrary ray-path parameter and $\bar{\sigma}$ an initial condition, for example, direction cosines. Then the inversion of the wave-vector and time transformations, followed by substitution into the coordinate space map determines the Lagrange manifold explicitly

$$
\bar{r}=\bar{r}(\gamma(\bar{p}, \omega), \bar{\sigma}(\bar{p}, \omega))=\nabla_{p} \Theta(\bar{p}, \omega) .
$$

Integration along the trajectories determines

$$
\Theta(\bar{p}, \omega)=\int_{\bar{p}_{0}}^{\bar{p}} \bar{r} \cdot d \bar{p}
$$

and hence, the phase

$$
\phi(\bar{r}, t, \bar{p}, \omega)=\bar{r} \cdot \bar{p}-\omega t-\Theta(\bar{p}, \omega) .
$$

We obtain a transport equation for the amplitudes by Taylor-expanding, the Hamiltonian near the Lagrange manifold

$$
H(\bar{r}, \bar{p})=H\left(\nabla_{p} \Theta, \bar{p}\right)+\left(\bar{r}-\nabla_{p} \Theta\right) \cdot \bar{D}=\left(\bar{r}-\nabla_{p} \Theta\right) \cdot \bar{D},
$$

where

$$
\bar{D}=\int_{0}^{1} \nabla_{r} H\left(\zeta\left(\bar{r}-\nabla_{p} \Theta\right)+\nabla_{p} \Theta, \bar{p}\right) d \zeta .
$$

Next, substituting (3.15) into (3.7), performing a partial integration and introducing the non-Hamiltonian flow

$$
\begin{aligned}
\overline{\dot{r}}=(\dot{x}, \dot{z}) & =\left(B+2 \omega m-3 U m^{2}-\frac{U}{4 H^{2} S}, \frac{2(\omega-U m) l}{S}\right), \\
\dot{t} & =-\left(m^{2}+\frac{l^{2}}{S}+\frac{1}{4 H^{2} S}\right), \quad \overline{\dot{p}}=-\bar{D},
\end{aligned}
$$


where the differentiations are with respect to the ray-path parameter, determines a transport equation in a neighborhood of the Lagrange manifold

$$
\begin{aligned}
\frac{d A_{k}}{d t} & -\nabla_{p} \cdot \bar{D} A_{k}+(3 U m-\omega) \frac{\partial^{2} A_{k-1}}{\partial x^{2}}+\frac{(U m-\omega)}{S} \frac{\partial A_{k-1}}{\partial z^{2}} \\
& +2\left(m \frac{\partial^{2} A_{k-1}}{\partial x \partial t}+\frac{1}{S} \frac{\partial^{2} A_{k-1}}{\partial z \partial t}+\frac{m l}{S} \frac{\partial^{2} A_{k-1}}{\partial z \partial x}\right)+\left(\frac{\partial}{\partial t}+U \frac{\partial}{\partial x}\right)\left(\frac{\partial^{2} A_{k-2}}{\partial x^{2}}+\frac{1}{S} \frac{\partial^{2} A_{k-2}}{\partial z^{2}}\right)=0 .
\end{aligned}
$$

The $k=0$ equation corresponds to equation (2.21) in [10]. With the amplitudes determined, $\psi(x, z, t)$ in (3.3) may now be determined at any point on the caustic curve through conventional asymptotic evaluation of the integral. A detailed treatment with an example appears in [2].

We conclude by noting that, in this approach the entire caustic curve, or locus of turning points, may be determined by setting the Hessian determinant of the phase to zero

$$
\operatorname{det}\left(\frac{\partial^{2} \phi}{\partial m \partial l}\right)=0
$$

Each real $\bar{p}=(m, l)$ satisfying this equation specifies a caustic point in wave-vector space. The corresponding point in configuration space may be obtained by substituting this wave-vector into the Lagrange manifold, (3.9). The locus of these configuration space points is the caustic curve.

4. Analysis I. Since the Lagrange manifold formalism is essentially an integral representation of the WKB approach, we may use it to study wave packet properties on the caustics in a manner analogous to that used by Yang to study wave packet properties away from the caustics. (The corresponding equations cited here appear in [10].)

By solving the eikonal equation for $\omega$, we obtain the same dispersion equation on the caustics that Yang determines away from the caustics, equation (see [10, equation (2.19)]),

$$
\omega=\sigma=U(z) m-\frac{B m}{K^{2}},
$$

where

$$
K^{2}=m^{2}+\frac{l^{2}}{S}+\frac{1}{4 H^{2} S} .
$$

(Yang uses $\sigma$ rather than $\omega$.) Further, from (3.12), we determine phase velocities

$$
C_{x}=\frac{\omega}{m}=U-\frac{B}{K^{2}}, \quad C_{z}=\frac{\omega}{l}=\frac{U m}{l}-\frac{B m}{l K^{2}}
$$

and group velocities

$$
C_{g x}=\frac{\partial \omega}{\partial m}=U(z)-\frac{\left(K^{2}-2 m^{2}\right) B}{K^{4}}, \quad C_{g z}=\frac{\partial \omega}{\partial l}=\frac{2 m l B}{S K^{4}}
$$

on the caustics identical to those determined by Yang away from the caustics, equations (see [10, equations (2.22)-(2.25)]). We note that, $C_{g x}$ and $C_{g z}$ also may be determined from Hamilton's equations if we replace the ray-path parameter $\gamma$ with $t$. Then, 
Hamilton's equations (cf. [10, equations (2.16) and (2.17)]) become

$$
\begin{aligned}
\frac{d x}{d t} & =\frac{\partial H / \partial m}{-\partial H / \partial \omega}=C_{g x}, \quad \frac{d z}{d t}=\frac{\partial H / \partial l}{-\partial H / \partial \omega}=C_{g z}, \\
\frac{d \sigma}{d t} & =\frac{\partial H / \partial \gamma}{-\partial H / \partial \omega}=0, \quad \frac{d m}{d t}=\frac{-\partial H / \partial x}{-\partial H / \partial \omega}=0, \\
\frac{d l}{d t} & =\frac{-\partial H / \partial z}{-\partial H / \partial \omega}=-m \frac{\partial U}{\partial z}-\frac{\left(1+4 H^{2} l^{2}\right) m B}{4 H^{3} S K^{4} \epsilon}-\frac{m}{K^{2}} \frac{\partial B}{\partial z}, \\
\frac{d t}{d t} & =\frac{-\partial H / \partial \omega}{-\partial H / \partial \omega}=1 .
\end{aligned}
$$

The equations for $d \sigma / d t, d m / d t$, and $d l / d t$ match Yang's equations (cf. [10, equations (2.26)-(2.28)]). We conclude by noting that the various simplifying approximations considered by Yang, for example, long planetary wave approximation, lead to corresponding simplifications in his modeling equations away from the caustics. Identical equations may be derived on the caustics. For brevity we do not present them here.

5. Potential vorticity equation in the general ocean circulation. The linearized nondimensional equation governing the potential vorticity in a stratified threedimensional quasi-geostrophic baroclinic ocean for large-scale motion can be written as

$$
\left(\frac{\partial}{\partial t}+U(x, y, z) \frac{\partial}{\partial x}+V(x, y, z) \frac{\partial}{\partial y}\right) q^{\prime}+B_{1} \frac{\partial \psi^{\prime}}{\partial x}-B_{2} \frac{\partial \psi^{\prime}}{\partial y}=0,
$$

where $q^{\prime}$ is the perturbation potential vorticity, defined as

$$
q^{\prime}=\frac{\partial^{2} \psi^{\prime}}{\partial x^{2}}+\frac{\partial^{2} \psi^{\prime}}{\partial y^{2}}+\frac{\partial}{\partial z}\left(\frac{1}{S} \frac{\partial \psi^{\prime}}{\partial z}\right)
$$

in the domain $\left(0 \leq x \leq x_{e}, 0 \leq y \leq 1,-1 \leq z \leq 0\right)$, where $x_{e}$ is the location of the eastern boundary. As above, $x, y$, and $z$ are the eastward, northward, and upward coordinates. $U(x, y, z)$ and $V(x, y, z)$ are the eastward and northward components of the mean ocean currents. $B_{1}$ and $B_{2}$ are, respectively, the meridional and zonal gradients of the mean potential vorticity, $Q$, that is,

$$
B_{1}=\frac{\partial Q}{\partial y}, \quad B_{2}=\frac{\partial Q}{\partial x}
$$

where

$$
Q=\beta y+\nabla^{2} \Phi+\frac{\partial}{\partial z}\left(\frac{1}{S} \frac{\partial \Phi}{\partial z}\right)
$$

$\nabla^{2}$ is the horizontal Laplacian operator, $S$ is the nondimensional stratification parameter defined above, and $\Phi$ is the stream function for the mean ocean current defined so that the wind-driven circulation satisfies the Sverdrup relation with the transport function

$$
\int_{z=-1}^{z=0} \Phi d z=-\frac{\partial \tau}{\partial y}\left(x_{e}-x\right)
$$


where $\tau$ is the zonal wind stress, specified so that the mean ocean circulation represents the subtropical ocean gyre. As above in (2.5), we assume that the vertical stratification parameter is exponential with scale $H$. We further assume that the vertical dependence of the mean zonal circulation takes the form

$$
F(z)=\frac{1}{A} e^{z / 2 H} \cos (k \pi z),
$$

where $k$ is the vertical wave number of the mean ocean circulation and $A$ is a normalization constant so that the vertically integrated transport is equal to the Sverdrup transport [11].

Analogous to the above, we begin by introducing a transformation

$$
\psi^{\prime}=e^{z / 2 H} \psi
$$

which allows us to rewrite the perturbation equation (5.1) as

$$
\left(\frac{\partial}{\partial t}+U \frac{\partial}{\partial x}+V \frac{\partial}{\partial y}\right) q+B_{1} \frac{\partial \psi}{\partial x}-B_{2} \frac{\partial \psi}{\partial x}=0
$$

where

$$
\begin{aligned}
q & =\frac{\partial^{2} \psi}{\partial x^{2}}+\frac{\partial^{2} \psi}{\partial y^{2}}+\frac{1}{S} \frac{\partial^{2} \psi}{\partial z^{2}}-\frac{\psi}{4 H^{2} S}, \\
B_{1} & =\frac{\partial Q}{\partial y}=\beta+\frac{1+4 H^{2} \pi^{2}\left(k^{2}+S\right)}{4 H^{2} S} U, \\
B_{2} & =\frac{\partial Q}{\partial x}=-\frac{1+4 H^{2} \pi^{2}\left(k^{2}+S\right)}{4 H^{2} S} V .
\end{aligned}
$$

The Lagrange manifold procedure developed above may be applied here to determine an asymptotic solution near the caustics. An integral solution of the form appearing in (3.2), (3.3), (3.4), and (3.5) is assumed, the only difference coming from the added dimension considered in coordinate space. Consequently, the amplitude $A$ and the phase $\phi$ are now functions of three coordinate space variables and their conjugate wave-vectors, that is, $\bar{r}=(x, y, z)$ then, $\bar{p}=(m, n, l)$, and hence, $d \bar{p}=d m d n d l$.

Then substituting the assumed integral solution into (5.7), introducing wave-vector and frequency, (cf. (3.6)), and following the procedure above leads to the Hamiltonian

$$
H(\bar{r}, \bar{p})=\left(m^{2}+n^{2}+\frac{l^{2}}{S}+\frac{1}{4 H^{2} S}\right)(\omega-U m-V n)+B_{1} m-B_{2} n .
$$

Proceeding through the algorithm, we obtain the transport equation

$$
\begin{aligned}
\frac{d A_{k}}{d t}- & \nabla \cdot \bar{D} A_{k}+(3 U m+V n-\omega) \frac{\partial^{2} A_{k-1}}{\partial x^{2}}+(3 V n+U m-\omega) \frac{\partial^{2} A_{k-1}}{\partial y^{2}} \\
& +\frac{(U m+V n-\omega)}{S} \frac{\partial^{2} A_{k-1}}{\partial z^{2}}+2\left(m \frac{\partial^{2} A_{k-1}}{\partial x \partial t}+n \frac{\partial^{2} A_{k-1}}{\partial y \partial t}+\frac{l}{S} \frac{\partial^{2} A_{k-1}}{\partial z \partial t}\right) \\
& +2(U m+V n) \frac{\partial^{2} A_{k-1}}{\partial x \partial y}+\frac{l}{S}\left(U \frac{\partial^{2} A_{k-1}}{\partial x \partial z}+V \frac{\partial^{2} A_{k-1}}{\partial y \partial z}\right) \\
& +\left(\frac{\partial}{\partial t}+U \frac{\partial}{\partial x}+V \frac{\partial}{\partial y}\right)\left(\frac{\partial^{2} A_{k-2}}{\partial x^{2}}+\frac{\partial^{2} A_{k-2}}{\partial y^{2}}+\frac{l}{S} \frac{\partial^{2} A_{k-2}}{\partial z^{2}}\right)=0
\end{aligned}
$$


where $\bar{D}$ is defined as in (3.16). The $k=0$ equation corresponds to equation (2.28) in [11].

6. Analysis II. We parallel the treatment above and begin by noting that if the eikonal equation is solved for $\omega$, we obtain Yang's dispersion equation (2.26) in [11],

$$
\omega=\sigma=U(x, y, z) m+V(y, z) n-\frac{B_{1} m}{K^{2}}-\frac{B_{2} n}{K^{2}},
$$

where

$$
K^{2}=m^{2}+n^{2}+\frac{l^{2}}{S}+\frac{1}{4 H^{2} S} .
$$

From (6.1), we determine identical phase velocities (cf. [11, equations (2.29)-(2.31)]),

$$
\begin{aligned}
& C_{x}=\frac{\omega}{m}=U+V \frac{n}{m}-\frac{B_{1}}{K^{2}}+\frac{n}{m K^{2}} B_{2}, \\
& C_{y}=\frac{\omega}{n}=U \frac{m}{n}+V-\frac{m}{n K^{2}} B_{1}+\frac{1}{K^{2}} B_{2}, \\
& C_{z}=\frac{\omega}{l}=U \frac{m}{l}+V \frac{n}{l}-\frac{m}{l K^{2}} B_{1}+\frac{n}{l K^{2}} B_{2},
\end{aligned}
$$

and group velocities (cf. [11, equations (2.32)-(2.34)]),

$$
\begin{aligned}
& C_{g x}=\frac{\partial \sigma}{\partial m}=U-\frac{\left(K^{2}-2 m^{2}\right) B_{1}+2 m n B_{2}}{K^{4}}, \\
& C_{g y}=\frac{\partial \sigma}{\partial n}=V+\frac{2 m n B_{1}+\left(K^{2}-2 n^{2}\right) B_{2}}{K^{4}}, \\
& C_{g z}=\frac{\partial \sigma}{\partial l}=\frac{2 l}{S K^{4}}\left(m B_{1}-n B_{2}\right),
\end{aligned}
$$

on the caustics to those determined away from the caustics.

In this setting, the corresponding Hamilton's equations become

$$
\begin{aligned}
\frac{d x}{d t} & =\frac{\partial H / \partial m}{-\partial H / \partial \omega}=C_{g x}, \quad \frac{d y}{d t}=\frac{\partial H / \partial n}{-\partial H / \partial \omega}=C_{g y}, \\
\frac{d z}{d t} & =\frac{\partial H / \partial l}{-\partial H / \partial \omega}=C_{g z}, \quad \frac{d \sigma}{d t}=\frac{\partial H / \partial y}{-\partial H / \partial \omega}=0, \\
\frac{d m}{d t} & =\frac{-\partial H / \partial x}{-\partial H / \partial \omega}=-m \frac{\partial U}{\partial x}-n \frac{\partial V}{\partial x}+\frac{m}{K^{2}} \frac{\partial B_{1}}{\partial x}-\frac{n}{K^{2}} \frac{\partial B_{2}}{\partial x}, \\
\frac{d n}{d t} & =\frac{-\partial H / \partial y}{-\partial H / \partial \omega}=-m \frac{\partial U}{\partial y}-n \frac{\partial V}{\partial y}+\frac{m}{K^{2}} \frac{\partial B_{1}}{\partial y}-\frac{n}{K^{2}} \frac{\partial B_{2}}{\partial y}, \\
\frac{d l}{d t} & =\frac{-\partial H / \partial z}{-\partial H / \partial \omega}=\frac{m}{K^{2}} \frac{\partial B_{1}}{\partial z}-\frac{n}{K^{2}} \frac{\partial B_{2}}{\partial z}-m \frac{\partial U}{\partial z}-n \frac{\partial V}{\partial z}-\frac{\left(1+4 H^{2} l^{2}\right)\left(m B_{1}-n B_{2}\right)}{4 H^{3} S K^{4} \epsilon}, \\
\frac{d t}{d t} & =\frac{-\partial H / \partial \omega}{-\partial H / \partial \omega}=1 .
\end{aligned}
$$

The equations for $d \sigma / d t, d m / d t, d n / d t$, and $d l / d t$ match Yang's equations (2.35)-(2.38) of [11]. Again, we conclude by noting that the various simplifying approximations considered by Yang, for example, long planetary wave approximation, 
lead to corresponding simplifications in his modeling equations away from the caustics. Identical equations may be derived on the caustics.

\section{REFERENCES}

[1] V. I. Arnol'd, On a characteristic class entering into conditions of quantization, Funkcional. Anal. i Priložen. 1 (1967), no. 1, 1-14 (Russian). MR 35\#2296. Zbl 175.20303.

[2] A. D. Gorman, On caustics associated with Rossby waves, Appl. Math. 41 (1996), no. 5, 321-328. MR 97h:76026. Zbl 870.34059.

[3] P. N. Ivanova and A. D. Gorman, On caustics associated with the linearized vorticity equation, Appl. Math. 43 (1998), no. 4, 255-262. MR 99d:76022. Zbl 938.34048.

[4] J. B. Keller, A geometrical theory of diffraction, Calculus of Variations and its Applications. Proceedings of Symposia in Applied Mathematics, vol. 8, For the American Mathematical Society: McGraw-Hill Book Co., Inc., New York, Toronto, London, 1958, pp. 27-52. MR 20\#640. Zbl 092.20604.

[5] P. D. Killworth, D. B. Chelton, and R. A. de Szoeke, The speed of observed and theoretical long extratropic planetary waves, J. Phys. Oceanogr. 27 (1997), 1946-1966.

[6] C. Knessl and J. B. Keller, Rossby waves, Stud. Appl. Math. 94 (1995), no. 4, 359-376. MR 96d:76017. Zbl 833.76014.

[7] V. P. Maslov, Theorie des Perturbationes et Methodes Asymptotique. Suivi de deux notes complementaires de V. I. Arnol'd et V. C. Bouslaev. Traduit par J. Lascoux et R. Seneor, Etudes mathematiques, Dunod, Gauthier-Villars, Paris, 1972 (French). Zbl 247.47010.

[8] J. Pedlosky, Geophysical Fluid Dynamics, 2nd ed., Springer-Verlag, New York, 1987. Zbl 713.76005 .

[9] H. J. Yang, Wave Packets and their Bifurcations in Geophysical Fluid Dynamics, Applied Mathematical Sciences, vol. 85, Springer-Verlag, New York, 1991. MR 92b:86005. Zbl 744.76010.

[10] _ Evolution of long planetary wave packets in a continuously stratified ocean, J. Phys. Oceanogr. 30 (2000a), 2111-2123.

[11]_, Long planetary wave packet regimes and complex climate variability in extratropical oceans, preprint, 2000b.

[12] __ Dynamics of long planetary wave packets in a continuously stratified ocean, Recent Research Developments in Physical Oceanography, Ed. S. G. Pandalai, Transworld Research Network, in press, 2000c.

Arthur D. Gorman: Department of Mathematics, Lafayette College, Easton, PA 18042, USA

E-mail address: gormana@1afayetta.edu

Huijun YANG: College of MARINe SCIENCE, University of SOUth Florida, St. Petersburg, FL 33701, USA

E-mail address: yang@marine.usf.edu 


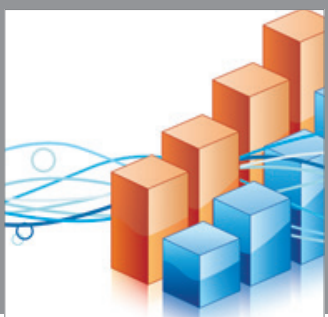

Advances in

Operations Research

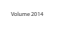

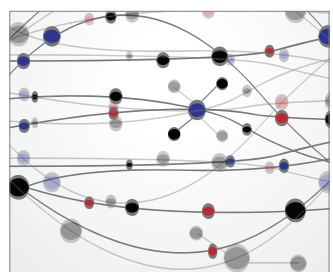

\section{The Scientific} World Journal
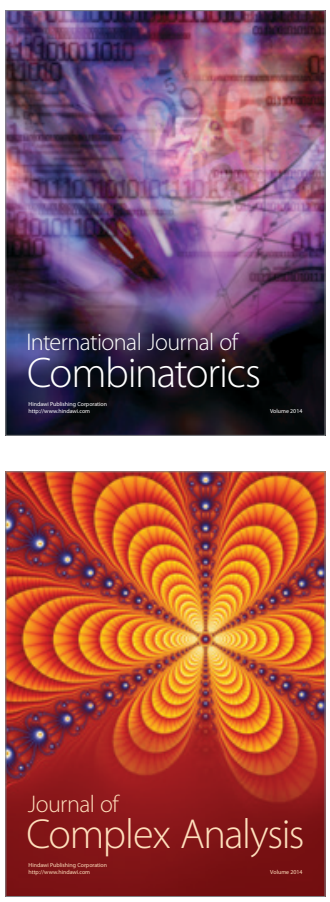

International Journal of

Mathematics and

Mathematical

Sciences
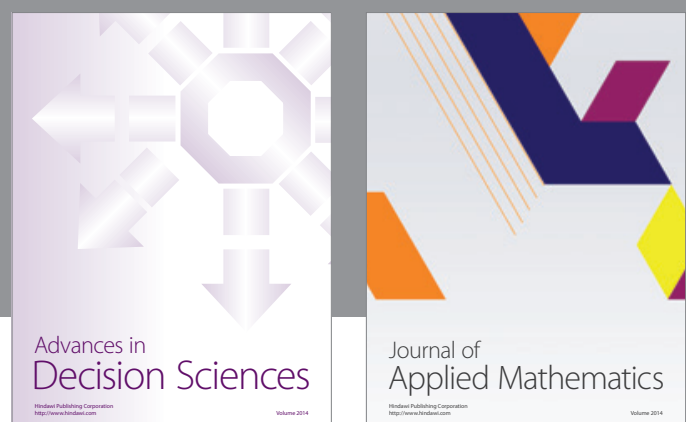

Journal of

Applied Mathematics
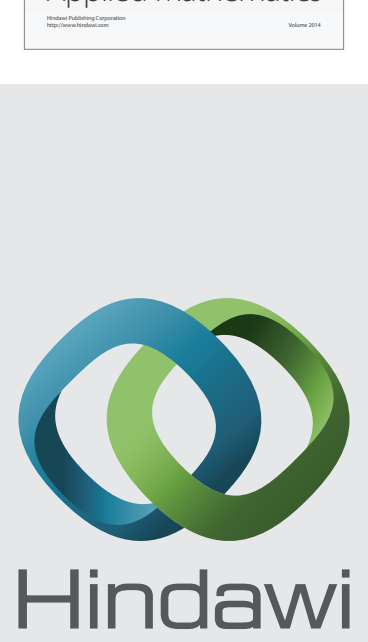

Submit your manuscripts at http://www.hindawi.com
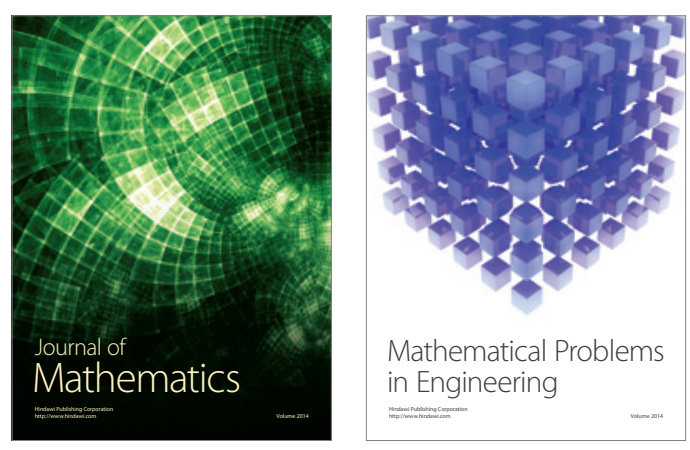

Mathematical Problems in Engineering
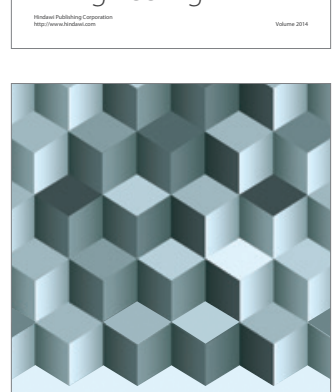

Journal of

Function Spaces
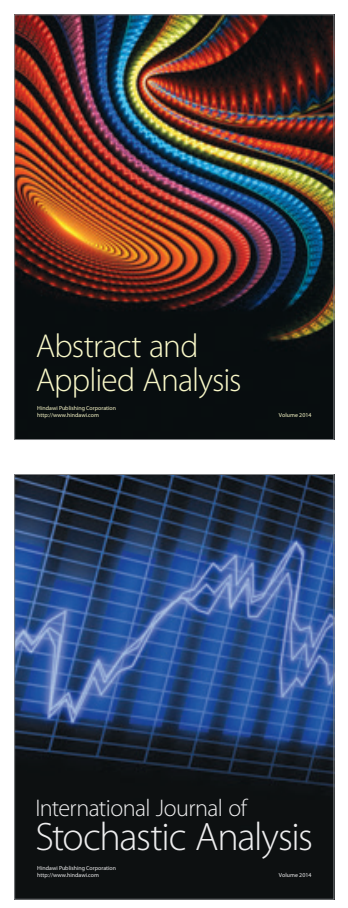

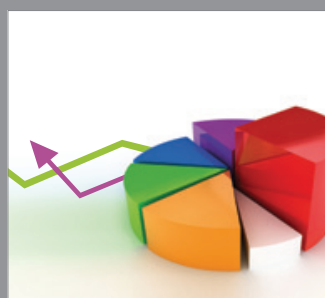

ournal of

Probability and Statistics

Promensencen
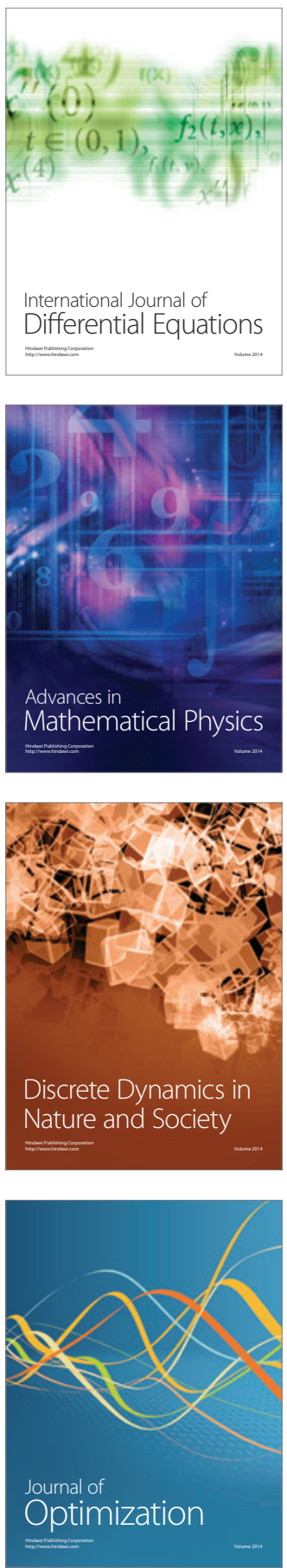